\title{
Urinary cadmium levels predict mortality of patients with acute heart failure
}

\author{
This article was published in the following Dove Press journal: \\ Therapeutics and Clinical Risk Management \\ 27 March 2017 \\ Number of times this article has been viewed
}

\section{Ching-Wei Hsu'-3 \\ Cheng-Hao Weng ${ }^{1-3}$ \\ Cheng-Chia Lee ${ }^{1-3}$ \\ Dan-Tzu Lin-Tan ${ }^{1-3}$ \\ Pao-Hsien Chu ${ }^{3,4}$ \\ Kuan-Hsing Chen ${ }^{1-3}$ \\ Tzung-Hai Yen ${ }^{1-3}$ \\ Wen-Hung Huang ${ }^{1-3}$}

'Department of Nephrology, Division of Clinical Toxicology, Chang Gung Memorial Hospital, Taipei, Taiwan, ROC; ${ }^{2}$ Department of Nephrology, Division of Clinical Toxicology, Linkou Medical Center, Taoyuan, Taiwan, ROC; ${ }^{3}$ Chang Gung University and School of Medicine, Taoyuan, Taiwan, ROC; ${ }^{4}$ Division of Cardiology, Chang Gung Memorial Hospital, Linkou Medical

Center, Taoyuan, Taiwan, ROC
Correspondence: Wen-Hung Huang Department of Nephrology, Division of Clinical Toxicology, Chang Gung Memorial Hospital 199, Tung-Hwa North Road, Taipei, Taiwan, ROC Tel +886 $3328|2008| 8 \mid$

Fax +88633288662

Email williammedia@gmail.com
Background: Acute heart failure (AHF) is a serious condition that is associated with increased mortality in critically ill patients. Previous studies indicated that environmental exposure to cadmium increases mortality of general populations. However, the relationship of cadmium exposure and mortality is unclear for AHF patients.

Materials and methods: A total of 153 patients with AHF in intensive care units (ICUs) met the inclusion criteria and were followed up for 6 months. Demographic data, AHF etiology, hematological and biochemical data, and hospital mortality were recorded. The scores of two predictive systems (Sequential Organ Failure Assessment [SOFA], Acute Physiology and Chronic Health Evaluation II [APACHE II]) for mortality in critically ill patients were calculated, and urinary cadmium levels were recorded.

Results: At the end of the follow-up period, the mortality rate was $24.8 \%$. The survivors ( $n=115$ ) had higher urinary cadmium levels on day 1 (D1UCd) of ICU admission than non-survivors $(n=38)$. A multiple linear regression analysis revealed a positive correlation between D1UCd and acute kidney injury, but a negative correlation between D1UCd and the level of serum albumin. A multivariate Cox analysis indicated that D1UCd was an independent predictor of mortality in AHF patients. For each increment of $1 \mu \mathrm{g}$ of D1UCd, the hazard ratio for ICU mortality was 1.20 (95\% confidence interval $[\mathrm{CI}]: 1.09-1.32, P<0.001)$. The area under the receiver operating characteristic curve for D1UCd was 0.84 (95\% CI: $0.78-0.91)$, better than the values for the SOFA and APACHE II systems.

Conclusion: The D1UCd may serve as a single predictor of hospital mortality for AHF patients in the ICU. Because of the high mortality and smaller sample size, more investigations are required to confirm these observations and elucidate the underlying mechanisms.

Keywords: acute heart failure, cadmium, intensive care unit, hospital mortality

\section{Introduction}

Acute heart failure (AHF) is a serious disease associated with high morbidity and mortality. ${ }^{1}$ When the symptoms of AHF are severe in the patients of intensive care units (ICUs), aggressive medical treatment and organ support are required. Only a few studies have examined the epidemiology of ICU patients with AHF, ${ }^{2,3}$ although some studies have examined specific aspects of such patients. ${ }^{4,5}$

Cadmium is a well-known toxic metal. ${ }^{6}$ Environmental and occupational exposure to cadmium is associated with several clinical diseases, including renal dysfunction, bone disease, and some cancers. ${ }^{6}$ The half-life period for cadmium is approximately 10 to 30 years and the absorbed cadmium is eliminated from the body primarily in urine. ${ }^{6}$ Because the urinary excretion of cadmium correlates well with cadmium accumulation in human bodies, the urinary cadmium levels may serve as an indicator of 
body cadmium burden. ${ }^{7}$ However, the correlation between urinary cadmium levels and mortality is uncertain in AHF patients.

In the past 20 years, several scoring systems have been used to evaluate illness severity and predict mortality for critically ill patients, such as the Sequential Organ Failure Assessment (SOFA), the Acute Physiology and Chronic Health Evaluation II (APACHE II), the Simplified Acute Physiology Score, and the Multiple Organ Dysfunction Score. ${ }^{8-11}$ However, these scoring systems are complicated and time-consuming in clinical practice, and there may be significant inter-observer variability among the physicians who calculate these scores. ${ }^{12}$ Moreover, there is still no single variable accurately predicting mortality in patients with AHF.

In this study, we evaluated the association between urinary cadmium levels and outcome of AHF patients and compared these results to the SOFA and APACHE II scoring systems.

\section{Methods}

This clinical study complied with the guidelines of the Declaration of Helsinki, and was approved by the Institutional Review Board (IRB) of Chang Gung Memorial Hospital, a tertiary referral center in Taiwan (IRB number: 101-3653C).

\section{Patient information and data collection}

All participants were at least 18 years old and admitted to the cardiac ICU or medical ICU. The included patients presented fatigue, limitation of physical activity, orthopnea and dyspnea, which were compatible with the typical symptoms of heart failure of the New York Heart Association Functional class III or IV. ${ }^{13}$ The diagnosis of AHF was based on a detailed taking of their history, the assessment of signs/ symptoms of congestion and/or hypoperfusion of organs, and confirmed by appropriate additional studies, including electrocardiography, chest roentgenogram and echocardiography, which were recommended by the guidelines of the American College of Cardiology/American Heart Association and the European Society of Cardiology. ${ }^{14,15}$ The exclusion criteria were as follows: total urine less than $300 \mathrm{~mL} /$ day on admission day 1; duration of ICU stay less than 1 day; duration of hospital stay more than 120 days; presence of end-stage renal disease; readmission to the ICU; history of occupational, residential, or other exposure to cadmium or history of intoxication from other heavy metals. In this study, acute respiratory failure was defined as acute onset of respiratory failure that required mechanical ventilator support. Acute kidney injury
(AKI) was defined as serum creatinine ( $\mathrm{SCr}$ ) above $2.0 \mathrm{mg} / \mathrm{dL}$ and/or daily urine amount less than $500 \mathrm{~mL}$.

For each patient we collected demographic data, laboratory data, length of stay in the ICU, pre-existing chronic disease, etiology of AHF, clinical condition upon ICU admission, data for scoring predictive indices (at least $12 \mathrm{~h}$ before death in non-survivors), and outcome. The blood samples were taken within $24 \mathrm{~h}$ after ICU admission. The urine samples were collected during the first $24 \mathrm{~h}$ to determine the total amount of urinary cadmium on day 1 (D1UCd) of ICU admission.

This study was approved by the Institutional Review Committee of our hospital and adhered to the principles of the Declaration of Helsinki. Written informed consent was obtained from every study patient.

\section{Clinical scoring systems}

The APACHE II and SOFA scoring models were used to assess the severity of illness for every enrolled participant. We calculated APACHE II scores as described previously; ${ }^{8}$ physiological assessments were performed using the highest abnormal physiological values obtained on day 1 of ICU admission. We evaluated SOFA scores as described by Vincent et al; ${ }^{11}$ the highest abnormal value was recorded for each of the six organ systems on day 1 of ICU admission.

\section{Measurement of urinary cadmium}

The urine samples of study patients were collected in cadmium-free bottles. The urinary cadmium levels were measured by the method proposed by Jin et al. ${ }^{16}$ Briefly, $100 \mu \mathrm{L}$ of urine and $500 \mu \mathrm{L}$ of trace-metal-grade distilled $0.8 \mathrm{M}$ HNO3 were added to a $1.5 \mathrm{~mL}$ Eppendorf vessel, which was shaken immediately. After overnight storage in a refrigerator, these vessels were permitted to warm to room temperature and whirl-mixed for 5-10 s. Then, the vessels were spun in an Eppendorf centrifuge for $5 \mathrm{~min}$ at 11,500 rpm. The clear supernatant samples were obtained after the above procedures and transferred directly to graphite furnace containers. We employed electrothermal atomic absorption spectrometry (SpectrAA-220 Zeeman; Varian, Palo Alto, CA, USA) to determine the cadmium levels in these samples. We confirmed the quality control consistently by internal and external quality control procedures. In addition, we used a certified commercially prepared product (Seronorm Trace Elements; Sero AS, Billingstad, Norway) to evaluate the intra-batch accuracy and ensure inter-batch standardization. The coefficient of variation for measurements of cadmium was $\leq 5.0 \%$. 


\section{Statistical analysis}

The Kolmogorov-Smirnov test was used to determine the normal distribution for continuous variables. Continuous variables are expressed as means \pm standard deviations or medians with interquartile ranges (IQRs), and categorical variables as numbers and percentages. Continuous variables were compared using the Student's $t$-test or Mann-Whitney $U$ test, as appropriate. Categorical variables were compared using the chi-squared test or Fisher's exact test. We used the linear regression model to identify factors associated with D1UCd. All potential variables $(P<0.05)$ in a simple linear regression model were entered into a multiple linear regression model with backward stepwise procedures. We used the Cox proportional hazard model to measure all variables and determine their significance for prediction of mortality. The hazard ratios (HRs) and 95\% confidence intervals (CIs) for death were obtained with this model. All potential variables $(P<0.05)$ in a univariate model were entered into a multivariate Cox model with forward stepwise procedures. We applied the Hosmer-Lemeshow (HL) test to evaluate the goodness-of-fit of these predictive models. ${ }^{17}$ We plotted receiver operating characteristic (ROC) curves and calculated the area under curve (AUC) to evaluate the performance of different predictors. ${ }^{18}$ The SPSS 18.0 for Windows XP (SPSS Inc., Chicago, IL,USA) software package was used in all analyses, and a two-sided $P$-value $<0.05$ was considered significant.

\section{Results}

\section{Patient characteristics}

A total of 153 critically ill patients with AHF (94 males and 59 females) were enrolled in this study (Table 1). The mean

Table I Baseline characteristics of acute heart failure patients on admission to the ICU $(n=I 53)$

\begin{tabular}{|c|c|c|c|}
\hline Characteristic & Survivor $(n=|| 5)$ & Non-survivor $(n=38)$ & $P$-value \\
\hline \multicolumn{4}{|l|}{ Demographics } \\
\hline Age (years) & $69.7 \pm 14.5$ & $71.3 \pm 13.1$ & 0.547 \\
\hline Sex (male) & $75(65.2)$ & $19(50.0)$ & 0.124 \\
\hline Smoking (yes) & $58(50.4)$ & II (28.9) & 0.024 \\
\hline BMI $\left(\mathrm{kg} / \mathrm{m}^{2}\right)$ & $23.6 \pm 4.4$ & $22.8 \pm 4.3$ & 0.373 \\
\hline ICU stay (days) & $6.0(4.0-13.0)$ & $20.0(9.5-28.5)$ & $<\mathbf{0 . 0 0 1}$ \\
\hline Hospital stay (days) & $19.0(12.0-35.0)$ & $29.5(17.0-39.3)$ & 0.243 \\
\hline \multicolumn{4}{|l|}{ Comorbidities } \\
\hline Previous CVD & $91(79.1)$ & $28(73.7)$ & 0.504 \\
\hline Previous CKD with abnormal SCr & $25(21.7)$ & $14(36.8)$ & 0.083 \\
\hline Previous CPD & $15(13.0)$ & $5(13.2)$ & 0.999 \\
\hline Hyperlipidemia & $38(33.0)$ & $10(26.3)$ & 0.546 \\
\hline Diabetes mellitus & $54(47.0)$ & $16(42.1)$ & 0.708 \\
\hline Hypertension & $73(66.1)$ & $20(52.6)$ & 0.255 \\
\hline \multicolumn{4}{|l|}{ Etiology of AHF } \\
\hline Ischemic heart disease & $72(62.6)$ & $26(68.4)$ & 0.564 \\
\hline Rheumatic heart disease & $12(10.4)$ & $2(5.3)$ & 0.519 \\
\hline Valvular heart disease & $9(7.8)$ & $4(10.5)$ & 0.737 \\
\hline Idiopathic DCM & $6(5.2)$ & I (2.6) & 0.682 \\
\hline COPD-related condition & $4(3.5)$ & I (2.6) & 0.999 \\
\hline Others & $12(10.4)$ & $4(10.5)$ & 0.999 \\
\hline \multicolumn{4}{|l|}{ ICU admission status } \\
\hline ARF due to pulmonary edema, with ventilators & $60(52.2)$ & $19(50.0)$ & 0.853 \\
\hline Cardiogenic shock, with inotropic drugs & $38(33.0)$ & $16(42.1)$ & 0.332 \\
\hline AMI & $29(25.2)$ & $18(47.4)$ & 0.015 \\
\hline AKI & $35(30.4)$ & $22(57.9)$ & 0.004 \\
\hline Others & $5(4.3)$ & I (2.6) & 0.999 \\
\hline \multicolumn{4}{|l|}{ Predictive indices } \\
\hline APACHE II score & $13.0(9.0-19.0)$ & $19.0(\mid 3.0-24.0)$ & 0.010 \\
\hline SOFA score & $5.0(3.0-7.0)$ & $6.0(3.8-9.0)$ & 0.044 \\
\hline DIUCd $(\mu g)$ & $0.73(0.39-1.53)$ & $3.42(1.34-6.75)$ & $<0.001$ \\
\hline
\end{tabular}

Notes: Data are presented as mean \pm standard deviations, numbers (percentages), or medians (interquartile ranges). CVDs included stroke, ischemic heart disease, valvular heart diseases, and peripheral vascular diseases. CKD was defined as persistent abnormal renal function (serum creatinine $>1.4 \mathrm{mg} / \mathrm{dL}$ ) for at least $6 \mathrm{months}$. CPDs included asthma, chronic bronchitis, COPD, and lung fibrosis. Hyperlipidemia was diagnosed by a physician and required regular treatments with antilipidemic agent. Diabetes mellitus was diagnosed by a physician and required regular treatments with antihyperglycemic drugs. Hypertension was defined as blood pressure above I40/90 mmHg based on at least two measurements and regular treatment with an antihypertensive drug. Shock was defined as mean arterial pressure less than $60 \mathrm{mmHg}$. Acute respiratory failure was defined as acute onset of respiratory failure that required ventilator support. AKI was defined as serum creatinine above $2.0 \mathrm{mg} / \mathrm{dL}$ and/or daily urine amount less than $500 \mathrm{~mL}$. Bold indicates clinical variables are significant in statistical analysis $(P<0.05)$.

Abbreviations: AHF, acute heart failure; AKI, acute kidney injury; AMI, acute myocardial infarction; APACHE, Acute Physiology and Chronic Health Evaluation; ARF, acute respiratory failure; BMI, body mass index; CKD, chronic kidney disease; CPD, chronic pulmonary disease; CVD, cardiovascular disease; DIUCd, day I urinary excretion of cadmium; DCM, dilated cardiomyopathy; ICU, intensive care unit; SCr, serum creatinine; SOFA, Sequential Organ Failure Assessment. 
Table 2 Baseline vital signs and biochemical data of acute heart failure patients on admission to the ICU $(n=153)$

\begin{tabular}{|c|c|c|c|}
\hline Variables & Survivor $(n=1 \mid 5)$ & Non-survivor $(n=38)$ & $P$-value \\
\hline \multicolumn{4}{|l|}{ Vital signs } \\
\hline Glasgow coma scale & $13.3 \pm 3.3$ & $12.4 \pm 3.8$ & 0.204 \\
\hline Mean arterial pressure & $87.9 \pm 20.0$ & $82.8 \pm 15.3$ & 0.115 \\
\hline Body temperature $\left({ }^{\circ} \mathrm{C}\right)$ & $36.9 \pm 0.9$ & $36.9 \pm 1.0$ & 0.806 \\
\hline Heart rate (beats $/ \mathrm{min}$ ) & $94.9 \pm 22.6$ & $98.8 \pm 23.7$ & 0.382 \\
\hline Respiration rate (times/min) & $20.5 \pm 4.7$ & $22.7 \pm 6.4$ & 0.054 \\
\hline \multicolumn{4}{|l|}{ Biochemical data } \\
\hline Arterial $\mathrm{pH}$ & $7.39 \pm 0.14$ & $7.38 \pm 0.14$ & 0.728 \\
\hline $\mathrm{PaO}_{2} / \mathrm{FiO}_{2}(\mathrm{mmHg})^{*}$ & $243.9(161.8-334.7)$ & $239.8(182.9-350.8)$ & 0.957 \\
\hline Alb $(g / d L)$ & $3.24 \pm 0.50$ & $2.95 \pm 0.5 \mathrm{I}$ & 0.005 \\
\hline AST (U/L) & $39.0(22.0-84.0)$ & $44.0(27.3-225.3)$ & 0.316 \\
\hline $\mathrm{ALT}(\mathrm{U} / \mathrm{L})$ & $29.0(15.5-59.0)$ & $29.5(15.0-56.0)$ & 0.850 \\
\hline Total bilirubin (mg/dL) & $0.8(0.6-1.6)$ & $0.8(0.5-1.3)$ & 0.832 \\
\hline BUN (mg/dL) & $31.0(19.0-52.0)$ & $57.5(28.8-74.8)$ & 0.002 \\
\hline $\mathrm{SCr}(\mathrm{mg} / \mathrm{dL})$ & $\mathrm{I} .4(\mathrm{I} . \mathrm{I}-2 . \mathrm{I})$ & $2.3(1.3-3.5)$ & 0.006 \\
\hline $\mathrm{Hgb}(\mathrm{g} / \mathrm{dL})$ & $11.9 \pm 2.9$ & $10.5 \pm 3.2$ & 0.002 \\
\hline WBC $\left(10^{3} / \mu \mathrm{L}\right)$ & $1 \mathrm{I} .2 \pm 4.0$ & $10.8 \pm 4.4$ & 0.691 \\
\hline Platelets $\left(10^{3} / \mu \mathrm{L}\right)$ & $201.8 \pm 88.0$ & $2|4.4 \pm 9| .7$ & 0.466 \\
\hline Cardiac ejection fraction (\%) & $35.0(26.0-49.0)$ & $37.5(24.5-45.8)$ & 0.737 \\
\hline
\end{tabular}

Note: ${ }^{*} \mathrm{PaO}_{2} / \mathrm{FiO}_{2}$ : ratio of arterial oxygen partial pressure to fraction of inspired oxygen. Data are presented as means \pm standard deviations or medians (interquartile ranges). Bold indicates clinical variables are significant in statistical analysis $(P<0.05)$.

Abbreviations: Alb, albumin; AST, aspartate aminotransferase; ALT, alanine aminotransferase; BUN, blood urea nitrogen; Hgb, hemoglobin; SCr, serum creatinine; WBC, white blood cells; ICU, intensive care unit.

patient age was $70.1 \pm 14.2$ years. The median duration of ICU stay was 7.0 days (IQR: 4.0-20.0), and the median duration of hospital stay was 21.0 days (IQR: 13.0-36.5). The median score of APACHE II was 15.0 (IQR: 10.0-20.0) and the median score of SOFA was 5.0 (IQR: $3.0-8.0$ ). The median D1UCd was $0.998 \mu \mathrm{g} /$ day (IQR: 0.475-2.287) and the mean D1UCd was $2.15 \pm 3.86 \mu \mathrm{g} /$ day. The main three etiologies were ischemic heart disease $(64.1 \%, n=98)$, rheumatic heart disease $(9.2 \%, \mathrm{n}=14)$, and valvular heart disease $(8.5 \%$, $\mathrm{n}=13)$. The initial status at ICU admission was as follows: $79(51.6 \%)$ patients had acute respiratory failure requiring mechanical ventilation due to acute pulmonary edema, $54(35.3 \%)$ had cardiogenic shock and received inotropic agents, 47 (30.7\%) had acute myocardial infarction (AMI) or unstable angina, and 57 (37.3\%) had AKI. The overall mortality rate was $24.8 \%(n=38)$. As shown in Tables 1 and 2 , the baseline characteristics and laboratory parameters of the survivors $(n=115)$ and non-survivors $(n=38)$ were compared. These results indicated that non-survivors had longer ICU stays, higher prevalence of AMI and AKI, higher APACHE II and SOFA scores, higher levels of D1UCd, and higher levels of blood urea nitrogen (BUN) and SCr. More of the survivors were smokers and survivors had higher levels of serum albumin (Alb) and hemoglobin ( $\mathrm{Hgb})$.

\section{Determinants of urinary cadmium excretion}

As shown in Table 3, simple linear regression analysis demonstrated D1UCd was positively related to AKI, but negatively related to the serum levels of Alb and Hgb. Stepwise multiple linear regression analysis indicated that D1UCd was positively related to AKI (Beta \pm standard error: $0.807 \pm 0.444$, $P=0.046$ ), but negatively related to the serum level of $\mathrm{Alb}$ (Beta \pm standard error: $-1.294 \pm 0.421, P=0.025$ ).

\section{Cox regression analysis for mortality}

Univariate Cox regression analysis indicated that AKI, high respiratory rate, high BUN, high APACHE II score, and high

Table 3 Determinants of urinary cadmium excretion in acute heart failure patients on day I of ICU admission $(n=153)$

\begin{tabular}{llll}
\hline Variable & $\begin{array}{l}\text { Simple linear regression } \\
(\boldsymbol{r})\end{array}$ & $\begin{array}{l}\boldsymbol{P} \text {-value } \\
\text { AKI (yes =I, no =0) }\end{array}$ & $\begin{array}{l}\text { Stepwise multiple linear regression } \\
\text { (Beta } \pm \text { standard error) }\end{array}$ \\
\hline $\mathrm{Alb}(\mathrm{g} / \mathrm{dL})$ & $0.2 \mathrm{II}$ & 0.009 & $0.807 \pm 0.444$ \\
$\mathrm{Hgb}(\mathrm{g} / \mathrm{dL})$ & -0.278 & 0.002 & $-1.294 \pm 0.42 \mathrm{I}$ \\
\hline
\end{tabular}

Abbreviations: ICU, intensive care unit; AKI, acute kidney injury; Alb, albumin; Hgb, hemoglobin. 
Table 4 Forward multivariate Cox analysis of risk factors for hospital mortality in acute heart failure patients on day I of ICU admission $(n=153)$

\begin{tabular}{lll}
\hline Variable & Hazard ratio (95\% CI) & P-value \\
\hline Respiration rate (each increment of & I.II (I.03-I.20) & 0.004 \\
I time/min) & & \\
DIUCd (each increment of I $\mu \mathrm{g})$ & $1.20(\mathrm{I} .09-1.32)$ & $<0.00 \mathrm{I}$ \\
\hline
\end{tabular}

Abbreviations: ICU, intensive care unit; $\mathrm{Cl}$, confidence interval; DIUCd, day I urinary excretion of cadmium.

D1UCd were potential predictors of mortality. As shown in Table 4, we used a multivariate Cox regression analysis with forward stepwise methods to determine the independent effects of these potential predictors. The results indicated that high respiratory rate and high D1UCd were independent predictors of ICU mortality. The HR of mortality for each increment of 1 time/min in respiratory rate was $1.11(95 \%$ CI: $1.03-1.20, P=0.004)$ and the HR of mortality for each increment of $1 \mu \mathrm{g} /$ day in urinary cadmium was $1.20(95 \%$ CI: $1.09-1.32, P<0.001)$.

\section{Goodness-of-fit and ROC curves of scoring systems and DIUCd}

The HL chi-square test was used to evaluate the goodnessof-fit and the results were obtained as follows: (1) D1UCd (HL chi-square $=8.32,8$ degrees of freedom $[d f], P=0.403$ );

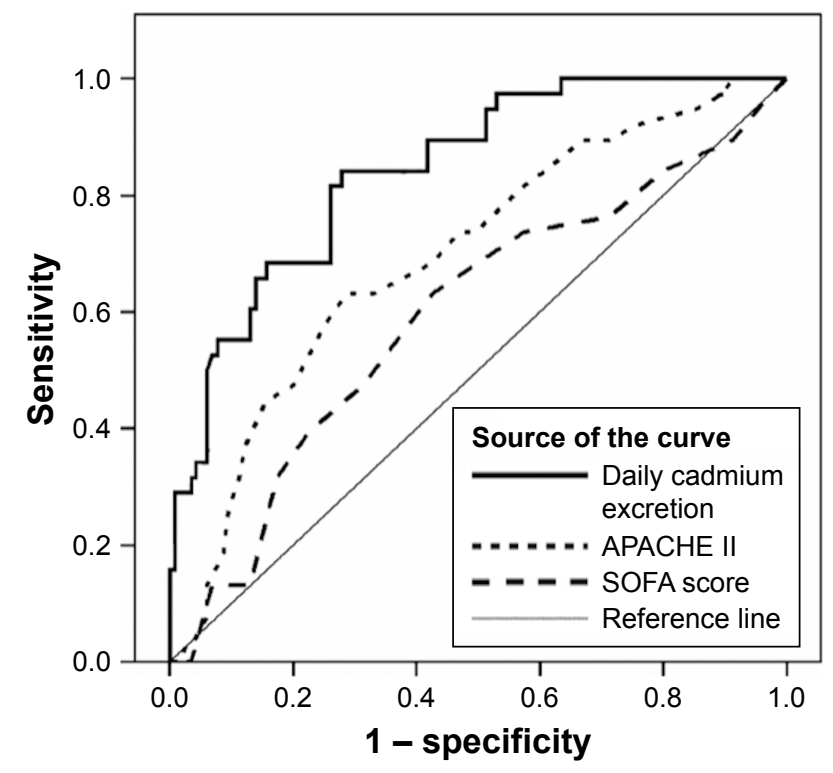

Figure I Receiver operating characteristic curves based on intensive care unit day I urinary cadmium excretion (AUC $=0.84 \pm 0.03,95 \% \mathrm{Cl}: 0.78-0.91$ ), APACHE II score (AUC $=0.70 \pm 0.05,95 \% \mathrm{Cl}: 0.60-0.77$ ), and SOFA score (AUC $=0.60 \pm 0.06,95 \% \mathrm{Cl}$ : 0.49-0.70).

Abbreviations: AUC, area under curve; $\mathrm{Cl}$, confidence interval; APACHE, Acute Physiology and Chronic Health Evaluation; SOFA, Sequential Organ Failure Assessment.
(2) APACHE II (HL chi-square $=5.93,8 d f, P=0.655$ ); (3) SOFA (HL chi-square $=3.84,7 d f, P=0.798$ ). As shown in Figure 1, the ROC curves were plotted for the two scoring models and D1UCd, and AUCs were also calculated. The results demonstrated that D1UCd (AUC $=0.84 \pm 0.03,95 \%$ CI: $0.78-0.91, P<0.001)$ had better discriminatory performance than the APACHE II score (AUC $=0.70 \pm 0.05,95 \%$ CI: $0.60-0.77, P<0.001)$ or the SOFA score (AUC $=0.60 \pm 0.06$, 95\% CI: $0.49-0.70, P=0.080$ ).

\section{Discussion}

This 6-month observational study demonstrated that the main cause of AHF in these critically ill patients was ischemic heart disease $(64.1 \%)$, similar to two previous studies of AHF patients $(70 \%$ and $61 \%))^{2,3}$ The mortality rate was $24.8 \%$, also similar to previous studies of ICU patients with AHF (30\% and 27.4\%). ${ }^{2,3}$ Notably, we found that D1UCd was remarkably related to ICU mortality after adjusting for potential variables, including APACHE II and SOFA scores. The HR of mortality for each increment of $1 \mu \mathrm{g} /$ day in urinary cadmium levels was 1.20. A review of the literature indicates this study is the first to reveal that urinary cadmium level was remarkably correlated with mortality of AHF patients.

There are several scoring models ${ }^{8-11}$ which are used to evaluate disease severity and predict the outcome of critically ill patients, including APACHE II and SOFA. Although these two models are widely applied in clinical practice, they are not user-friendly systems. In addition, such models are subject to significant inter-observer differences. ${ }^{19}$ Therefore, a single, reliable, and unbiased predictor of mortality is needed for critical patients because these patients often require prompt and aggressive treatments. Based on the study results, we found that the D1UCd is a simple predictor of mortality for critically ill AHF patients.

The general population (without occupational exposure to cadmium) has a urinary excretion of cadmium less than $2 \mu \mathrm{g} / \mathrm{day},{ }^{20}$ but there are no similar data of urinary cadmium levels in AHF patients, after reviewing the previous reports. In this study, the mean D1UCd of critically ill AHF patients was $2.15 \pm 3.86 \mu \mathrm{g} / \mathrm{day}$, which was higher than that $(1.07 \pm 1.45 \mu \mathrm{g} / \mathrm{day})^{21}$ of the general population in Taiwan. Although the physiological mechanism underlying the connection between elevated cadmium levels in biologic fluids and heart failure is unknown, several studies provide some insight. In rats, the heart muscle cells could be deformed by cadmium poisoning because of the increment of free radicals and lipid peroxidation, based on ultrastructural examination. ${ }^{22}$ Another rat study indicated that cadmium acted 
solely as an inhibitor of heart pyruvate-malate-supported mitochondrial respiration, suggesting that disturbances in myocardial metabolism and function may occur following cadmium exposure. ${ }^{23}$ Furthermore, an epidemiologic study of 12,049 US participants demonstrated that urinary cadmium levels were significantly correlated with self-reported prevalence of heart failure. ${ }^{24}$ A prospective cohort study of 3,348 adults in the US also revealed a significant association between urine cadmium and increased incidence of heart failure. ${ }^{25}$ Meanwhile, a study of 4,378 participants in Sweden with a 17-year follow-up period demonstrated that the participants in the fourth quartile of blood cadmium levels had significantly higher incidence of heart failure than those in the first quartile. ${ }^{26}$ All of these findings suggest that elevated cadmium levels may exacerbate the critical condition and increase hospital mortality in AHF patients. However, further investigations are needed to identify the mechanism and confirm the causal effect.

In general, AUC lower than 0.7 was considered as an indicator of poor performance, and higher than 0.8 as good performance. The analytic results indicated that D1UCd outperformed the APACHE II and SOFA scoring models and had the highest AUC $(0.84 \pm 0.03$, 95\% CI: 0.78-0.91). We also used the HL goodness-of-fit test to evaluate the calibration of models. Generally, HL statistics lower than 15 and $P$-values of 0.2 to 0.8 indicate a good fit. ${ }^{27,28}$ The study results showed D1UCd had a good calibration (HL chi-square $=8.32$, $P=0.403$ ). These findings revealed that D1UCd had a high discriminatory power and good calibration as a predictor of mortality for AHF patients, and was better than the established predictive scoring systems. Therefore, D1UCd may serve as a single predictor of mortality for AHF patients. It remains to be demonstrated whether lowering the body cadmium levels of AHF patients by chelation therapy will reduce their disease severity and improve their survival rate.

Patients admitted for AHF often develop exacerbated clinical renal function, ${ }^{29}$ which is mediated through several mechanisms, including renal hypoperfusion caused by low cardiac output, elevated central venous pressure, activation of renin-angiotensin-aldosterone system, endothelial dysfunction, and others. ${ }^{29}$ Moreover, some drugs for treating AHF and AMI are also associated with impairment of renal function, especially loop diuretics. ${ }^{29}$ In addition, hypoalbuminemia is common in patients with $\mathrm{AHF},{ }^{30}$ which may result from enhanced transcapillary escape rate of Alb from the intravascular to extravascular space, loss of nutrients due to increased hepatic venous congestion, decreased synthesis of Alb by liver and protein-losing enteropathy. ${ }^{30}$ Furthermore, AKI itself would accelerate protein breakdown, which is attributed to acute disease process, uremic toxins, inflammatory mediators, metabolic acidosis, hormonal imbalance and other factors, resulting in hypoalbuminemia. ${ }^{31}$ In the current study, we demonstrated D1UCd was positively correlated with AKI, but negatively correlated with serum Alb level in AHF patients. Cadmium exposure has well-known toxic effects on the kidneys ${ }^{16}$ and severe AHF may induce AKI in critically ill patients, so higher urinary cadmium levels might be observed in this population. Moreover, the increased cadmium levels in biologic fluids may influence the synthesis and metabolism of Alb, based on several in vitro studies. For example, Wan et $\mathrm{al}^{32}$ found lower Alb production in rat hepatocytes treated with cadmium and these perturbations were not alleviated after the cells were returned to cadmium-free medium. Gena et $\mathrm{a}^{33}$ reported that cadmium can lead to albuminuria in a pig proximal tubular cell model by impairing reabsorption and secretion. However, further studies are needed to explore the causal relationships between cadmium, AKI, and serum Alb in patients with AHF.

Cadmium has a long biological half-life (10-30 years) in humans and mainly accumulates in the liver and kidney. ${ }^{6,34}$ After uptake from the lung or the gastrointestinal tract, cadmium in blood is bound to Alb and high molecular weight proteins and transported to the liver, ${ }^{35}$ where it is bound to metallothionein. After a few days of cadmium exposure, cadmium-metallothionein compounds appear in the blood. ${ }^{35}$ Because of the small molecular size, these compounds are efficiently filtered by the glomeruli and reabsorbed by the proximal tubule cells. ${ }^{6,35,36}$ Then, the absorbed cadmium accumulates in the kidneys for a long time with a low rate of urinary excretion. ${ }^{6}$ Although cadmium exposure is a risk factor of cardiovascular diseases in humans, ${ }^{25,26}$ and may disturb myocardial metabolism (suggested by animal studies), ${ }^{22,23}$ it is uncertain whether the basic metabolism of cadmium would be changed in patients with cardiac diseases, including heart failure. Hence, further studies are needed to elucidate its specific role in AHF patients.

Cigarettes are one of the most prominent sources of cadmium exposure, and are associated with an increased mortality due to cardiovascular diseases. ${ }^{6,37}$ Cadmium oxide produced during the burning of tobacco has a high bioavailability in humans. ${ }^{38}$ Approximately $30 \%-40 \%$ of the inhaled cadmium oxide is absorbed into the blood circulatory system. ${ }^{38}$ Compared with non-smokers, smokers have 4-5 times higher cadmium levels in their blood and 2-3 times higher contents of cadmium in their kidneys. ${ }^{38}$ Although previous studies demonstrated that smoking was a well-known risk factor of mortality and significantly correlated with blood cadmium level, we observed that survivors were more likely to be smokers than non-survivors 
$(50.4 \%$ vs $28.9 \%, P=0.024)$ in these AHF patients. In addition, we found that D1UCd remained independently associated with increased mortality after adjustment for smoking in this study, which indicated that other causes except smoking may be the sources of cadmium exposure in these study patients. Since patients with history of occupational exposure to cadmium or history of intoxication from other heavy metals were excluded, the source of cadmium is most likely from the exposure in daily life such as contaminated food, drinking water, or passive smoking. Further studies are required to explore whether reducing cadmium exposure reduces the mortality of AHF patients.

There are several limitations of this study. First, although D1UCd was significantly associated with hospital mortality in critical AHF patients, the causal relationship is uncertain. Future investigations are required to clarify the underlying mechanisms. Second, not having serial assessments of B-type natriuretic peptide (BNP) is a limitation because a BNP immunoassay was not available to us at the time of this study. BNP reflects cardiac structure and function and can assist in the diagnosis of AHF. However, whether BNP can predict short-term outcome of AHF remains unclear. ${ }^{39}$ Third, due to the fact that this study was conducted at a single institution with a heterogeneous group of subjects, the study results may not apply to other hospitals. Hence, a multi-center investigation with more patients is warranted to verify the current findings in the future.

\section{Conclusion}

This is the first study to demonstrate that the D1UCd was significantly and independently associated with hospital mortality of critical AHF patients. Notably, the D1UCd has good calibration and discriminatory power, and outperformed the established predictive scoring systems. Moreover, D1UCd is a single variable with minimal inter-observer differences. Therefore, D1UCd should be considered a good predictor of mortality for critical AHF patients. Further investigations are needed to clarify these observations.

\section{Disclosure}

The authors report no conflicts of interest in this work.

\section{References}

1. Siirilä-Waris K, Lassus J, Melin J, Peuhkurinen K, Nieminen MS, Harjola VP; FINN-AKVA Study Group. Characteristics, outcomes, and predictors of 1-year mortality in patients hospitalized for acute heart failure. Eur Heart J. 2006;27(24):3011-3017.

2. Rudiger A, Businger F, Streit M, Schmid ER, Maggiorini M, Follath F. Presentation and outcome of critically ill medical and cardiac-surgery patients with acute heart failure. Swiss Med Wkly. 2009;139(7-8): 110-116.
3. Zannad F, Mebazaa A, Juilliere Y, et al. Clinical profile, contemporary management and one-year mortality in patients with severe acute heart failure syndromes: The EFICA study. Eur J Heart Fail. 2006;8(7): 697-705.

4. Nieminen MS, Brutsaert D, Dickstein K, et al. EuroHeart Failure Survey II (EHFS II): a survey on hospitalized acute heart failure patients: description of population. Eur Heart J. 2006;27(22):2725-2736.

5. Yancy CW, Lopatin M, Stevenson LW, De Marco T, Fonarow GC; ADHERE Scientific Advisory Committee and Investigators. Clinical presentation, management, and in-hospital outcomes of patients admitted with acute decompensated heart failure with preserved systolic function: a report from the Acute Decompensated Heart Failure National Registry (ADHERE) Database. J Am Coll Cardiol. 2006; 47(1):76-84.

6. Järup L, Berglund M, Elinder CG, Nordberg G, Vahter M. Health effects of cadmium exposure - a review of the literature and a risk estimate. Scand J Work Environ Health. 1998;24 Suppl 1:1-51.

7. Buchet JP, Lauwerys R, Roels H, et al. Renal effects of cadmium body burden of the general population. Lancet. 1990;336(8717):699-702.

8. Knaus WA, Draper EA, Wagner DP, Zimmerman JE. APACHE II: a severity of disease classification system. Crit Care Med. 1985;13(10): 818-829.

9. Le Gall JR, Lemeshow S, Saulnier F. A new Simplified Acute Physiology Score (SAPS II) based on a European/North American multicenter study. JAMA. 1993;270(24):2957-2963.

10. Marshall JC, Cook DJ, Christou NV, Bernard GR, Sprung CL, Sibbald WJ. Multiple organ dysfunction score: a reliable descriptor of a complex clinical outcome. Crit Care Med. 1995;23(10):1638-1652.

11. Vincent JL, Moreno R, Takala J, et al. The SOFA (Sepsis-related Organ Failure Assessment) score to describe organ dysfunction/failure. On behalf of the Working Group on Sepsis-Related Problems of the European Society of Intensive Care Medicine. Intensive Care Med. 1996; 22(7):707-710.

12. Polderman KH, Girbes AR, Thijs LG, Strack van Schijndel RJ. Accuracy and reliability of APACHE II scoring in two intensive care units. Problems and pitfalls in the use of APACHE II and suggestions for improvement. Anaesthesia. 2001;56(1):47-50.

13. Bennett JA, Riegel B, Bittner V, Nichols J. Validity and reliability of the NYHA classes for measuring research outcomes in patients with cardiac disease. Heart Lung. 2002;31(4):262-270.

14. Hunt SA; American College of Cardiology; American Heart Association Task Force on Practice Guidelines (Writing Committee to Update the 2001 Guidelines for the Evaluation and Management of Heart Failure). ACC/AHA 2005 guideline update for the diagnosis and management of chronic heart failure in the adult: a report of the American College of Cardiology/American Heart Association Task Force on Practice Guidelines (writing committee to update the 2001 guidelines for the evaluation and management of heart failure). J Am Coll Cardiol. 2005; 46(6):e1-e82.

15. Nieminen MS, Bohm M, Cowie MR, et al. Executive summary of the guidelines on the diagnosis and treatment of acute heart failure: the Task Force on Acute Heart Failure of the European Society of Cardiology. Eur Heart J. 2005;26(4):384-416.

16. Jin $\mathrm{T}$, Nordberg $\mathrm{M}$, Frech $\mathrm{W}$, et al. Cadmium biomonitoring and renal dysfunction among a population environmentally exposed to cadmium from smelting in China (ChinaCad). Biometals. 2002;15(4):397-410.

17. Lemeshow S, Hosmer DW Jr. A review of goodness of fit statistics for use in the development of logistic regression models. Am J Epidemiol. 1982;115(1):92-106.

18. Hanley JA, McNeil BJ. The meaning and use of the area under a receiver operating characteristic (ROC) curve. Radiology. 1982; 143(1):29-36.

19. Polderman KH, Jorna EM, Girbes AR. Inter-observer variability in APACHE II scoring: effect of strict guidelines and training. Intensive Care Med. 2001;27(8):1365-1369.

20. Bernard A, Lauwerys R. Effects of cadmium exposure in humans. In: Foulkes EC, editor. Handbook of experimental pharmacology. 1986;80:135-177. 
21. Lin JL, Lu FH, Yeh KH. Increased body cadmium burden in Chinese women without smoking and occupational exposure. J Toxicol Clin Toxicol. 1995;33(6):639-644.

22. Ozturk IM, Buyukakilli B, Balli E, Cimen B, Gunes S, Erdogan S. Determination of acute and chronic effects of cadmium on the cardiovascular system of rats. Toxicol Mech Methods. 2009;19(4):308-317.

23. Kisling GM, Kopp SJ, Paulson DJ, Hawley PL, Tow JP. Inhibition of rat heart mitochondrial respiration by cadmium chloride. Toxicol Appl Pharmacol. 1987;89(3):295-304.

24. Peters JL, Perlstein TS, Perry MJ, McNeely E, Weuve J. Cadmium exposure in association with history of stroke and heart failure. Environ Res. 2010;110(2):199-206.

25. Tellez-Plaza M, Guallar E, Howard BV, et al. Cadmium exposure and incident cardiovascular disease. Epidemiology. 2013;24(3):421-429.

26. Borne Y, Barregard L, Persson M, Hedblad B, Fagerberg B, Engstrom G. Cadmium exposure and incidence of heart failure and atrial fibrillation: a population-based prospective cohort study. BMJ Open. 2015; 5(6): e007366.

27. Angus DC, Clermont G, Kramer DJ, Linde-Zwirble WT, Pinsky MR. Short-term and long-term outcome prediction with the Acute Physiology and Chronic Health Evaluation II system after orthotopic liver transplantation. Crit Care Med. 2000;28(1):150-156.

28. Rosenberg AL. Recent innovations in intensive care unit risk-prediction models. Curr Opin Crit Care. 2002;8(4):321-330.

29. Carubelli V, Metra M, Lombardi C, et al. Renal dysfunction in acute heart failure: epidemiology, mechanisms and assessment. Heart Fail Rev. 2012;17(2):271-282.

30. Uthamalingam S, Kandala J, Daley M, et al. Serum albumin and mortality in acutely decompensated heart failure. Am Heart J. 2010; 160(6):1149-1155.
31. Wiedermann CJ, Wiedermann W, Joannidis M. Hypoalbuminemia and acute kidney injury: a meta-analysis of observational clinical studies. Intensive Care Med. 2010;36(10):1657-1665.

32. Wan X, Lachapelle M, Marion M, Fournier M, Denizeau F. Recovery potential of hepatocytes from inhibition of albumin secretion by cadmium. J Toxicol Environ Health. 1993;38(4):381-392.

33. Gena $\mathrm{P}$, Calamita G, Guggino WB. Cadmium impairs albumin reabsorption by down-regulating megalin and $\mathrm{ClC} 5$ channels in renal proximal tubule cells. Environ Health Perspect. 2010;118(11):1551-1556.

34. Järup L. Cadmium overload and toxicity. Nephrol Dial Transplant. 2002;17 Suppl 2:35-39.

35. Nordberg M. General aspects of cadmium: transport, uptake and metabolism by the kidney. Environ Health Perspect. 1984;54:13-20.

36. Tellez-Plaza M, Jones MR, Dominguez-Lucas A, Guallar E, Navas-Acien A. Cadmium exposure and clinical cardiovascular disease: a systematic review. Curr Atheroscler Rep. 2013;15(10):356.

37. Hsu CW, Yen TH, Chen KH, et al. Effect of blood cadmium level on mortality in patients undergoing maintenance hemodialysis. Medicine (Baltimore). 2015;94(42):e1755.

38. Satarug S, Moore MR. Adverse health effects of chronic exposure to low-level cadmium in foodstuffs and cigarette smoke. Environ Health Perspect. 2004;112(10):1099-1103.

39. Carpenter CR, Keim SM, Worster A, Rosen P, BEEM (Best Evidence in Emergency Medicine). Brain natriuretic peptide in the evaluation of emergency department dyspnea: is there a role? J Emerg Med. 2012; 42(2):197-205
Therapeutics and Clinical Risk Management

\section{Publish your work in this journal}

Therapeutics and Clinical Risk Management is an international, peerreviewed journal of clinical therapeutics and risk management, focusing on concise rapid reporting of clinical studies in all therapeutic areas outcomes, safety, and programs for the effective, safe, and sustained use of medicines. This journal is indexed on PubMed Central, CAS,

\section{Dovepress}

EMBase, Scopus and the Elsevier Bibliographic databases. The manuscript management system is completely online and includes a very quick and fair peer-review system, which is all easy to use. Visit http://www.dovepress.com/testimonials.php to read real quotes from published authors. 